\title{
GSVA: gene set variation analysis for microarray and RNA-Seq data
}

\author{
Sonja Hänzelmann ${ }^{1,2}$, Robert Castelo ${ }^{1,2^{*}}$ and Justin Guinney ${ }^{3^{*}}$
}

\begin{abstract}
Background: Gene set enrichment (GSE) analysis is a popular framework for condensing information from gene expression profiles into a pathway or signature summary. The strengths of this approach over single gene analysis include noise and dimension reduction, as well as greater biological interpretability. As molecular profiling experiments move beyond simple case-control studies, robust and flexible GSE methodologies are needed that can model pathway activity within highly heterogeneous data sets.

Results: To address this challenge, we introduce Gene Set Variation Analysis (GSVA), a GSE method that estimates variation of pathway activity over a sample population in an unsupervised manner. We demonstrate the robustness of GSVA in a comparison with current state of the art sample-wise enrichment methods. Further, we provide examples of its utility in differential pathway activity and survival analysis. Lastly, we show how GSVA works analogously with data from both microarray and RNA-seq experiments.

Conclusions: GSVA provides increased power to detect subtle pathway activity changes over a sample population in comparison to corresponding methods. While GSE methods are generally regarded as end points of a bioinformatic analysis, GSVA constitutes a starting point to build pathway-centric models of biology. Moreover, GSVA contributes to the current need of GSE methods for RNA-seq data. GSVA is an open source software package for R which forms part of the Bioconductor project and can be downloaded at http://www.bioconductor.org.
\end{abstract}

\section{Background}

The ability to measure mRNA abundance at a genomic scale has led to many efforts to catalog the diverse molecular patterns underlying biological processes. To facilitate the interpretation and organization of long lists of genes resulting from microarray experiments, gene set enrichment (GSE) methods have been introduced. They systematically measure and annotate molecular profiles that are inherently noisy and difficult to interpret. GSE analyses begin by obtaining a ranked gene list, typically derived from a microarray experiment that studies gene expression changes between two groups. The genes are then mapped into predefined gene sets and their gene expression statistic is summarized into a single enrichment score for each gene set. A significant benefit of these pathway-based methods is interpretability: gene function

*Correspondence: robert.castelo@upf.edu; justin.guinney@sagebase.org 1 Research Program on Biomedical Informatics (GRIB), Hospital del Mar Medical Research Institute (IMIM), Barcelona, Catalonia, Spain

3 Sage Bionetworks, 1100 Fairview Ave N., Seattle, Washington, 98109 USA Full list of author information is available at the end of the article is collectively exerted and may vary by environmental stimuli, genetic modifications, or disease state. Thus, organizing genes into gene sets provides a more intuitive and stable context for assessing biological activity.

Many methodological variations of GSE methods have been proposed [1-6], including non-parametric enrichment statistics $[4,7]$, battery testing [8-10], and focused gene set testing $[1,11,12]$. Battery testing methods aim at identifying gene sets standing out from a large collection of annotated pathways and gene signatures. Focused gene set testing methods try to carefully evaluate a few gene sets that are relevant to the experiment being analyzed [12]. GSE methods have been successfully applied in many experimental conditions to interpret the pathway architecture of biological states including cancer $[13,14]$, metabolic disease [15], and development [16]. For a recent review on GSE methods the reader may consult [17].

An important distinction among many of the GSE methods is the definition of the null hypothesis that is tested [18]. The null hypothesis of a competitive test declares 
that there are no differences between genes inside and outside the gene set (e.g., [4]). A self-contained test defines its null hypothesis only in terms of the genes inside the gene set being tested (e.g., [1]). More concretely, for a self-contained test on a gene set, the differential expression of just one of its genes allows one to reject the null hypothesis of no differential expression for that gene set. It follows, that self-contained tests provide higher power than competitive tests to detect subtle changes of expression in a gene set. But they may not be useful to single out a few gene sets in a battery testing setting because of the potentially large number of reported results.

Finally, many GSE methods assume two classes (e.g. case/control) and evaluate enrichment within this context [19-22]. The limits imposed by this assumption become evident with the rise of large genomic studies, such as The Cancer Genome Atlas project (TCGA - http:// cancergenome.nih.gov), an ambitious project with the goal to identify the molecular determinants of multiple cancer types. In contrast to case-control studies with small sample sizes, the TCGA project has large patient cohorts with multiple phenotypes, structured with hierarchical, multi-class, and censored data. Hence, GSE methods are needed that can assess pathway variation across large, heterogeneous populations with complex phenotypic traits.

To address these challenges, we present a nonparametric, unsupervised method called Gene Set Variation Analysis (GSVA). GSVA calculates sample-wise gene set enrichment scores as a function of genes inside and outside the gene set, analogously to a competitive gene set test. Further, it estimates variation of gene set enrichment over the samples independently of any class label. Conceptually, this methodology can be understood as a change in coordinate systems for gene expression data, from genes to gene sets. This transformation facilitates post-hoc construction of pathway-centric models, such as differential pathway activity identification or survival prediction. Further, we demonstrate the flexibility of GSVA by applying it to RNA-seq data.

\section{Implementation}

A schematic overview of the GSVA method is provided in Figure 1, which shows the two main required inputs: a matrix $X=\left\{x_{i j}\right\}_{p \times n}$ of normalized expression values (see Methods for details on the preprocessing steps) for $p$ genes by $n$ samples, where typically $p \gg n$, and a collection of gene sets $\Gamma=\left\{\gamma_{1}, \ldots, \gamma_{m}\right\}$. We shall denote by $x_{i}$ the expression profile of the $i$-th gene, by $x_{i j}$ the specific expression value of the $i$-th gene in the $j$-th sample, and by $\gamma_{k}$ the subset of row indices in $X$ such that $\gamma_{k} \subset\{1, \ldots p\}$ defines a set of genes forming a pathway or some other functional unit. Let $\left|\gamma_{k}\right|$ be the number of genes in $\gamma_{k}$.

GSVA starts by evaluating whether a gene $i$ is highly or lowly expressed in sample $j$ in the context of the sample population distribution. Probe effects can alter hybridization intensities in microarray data such that expression values can greatly differ between two non-expressed genes [23]. Analogous gene-specific biases, such as GC content or gene length have been described in RNA-seq data [24]. To bring distinct expression profiles to a common scale, an expression-level statistic is calculated as follows. For each gene expression profile $x_{i}=\left\{x_{i 1}, \ldots, x_{i n}\right\}$, a

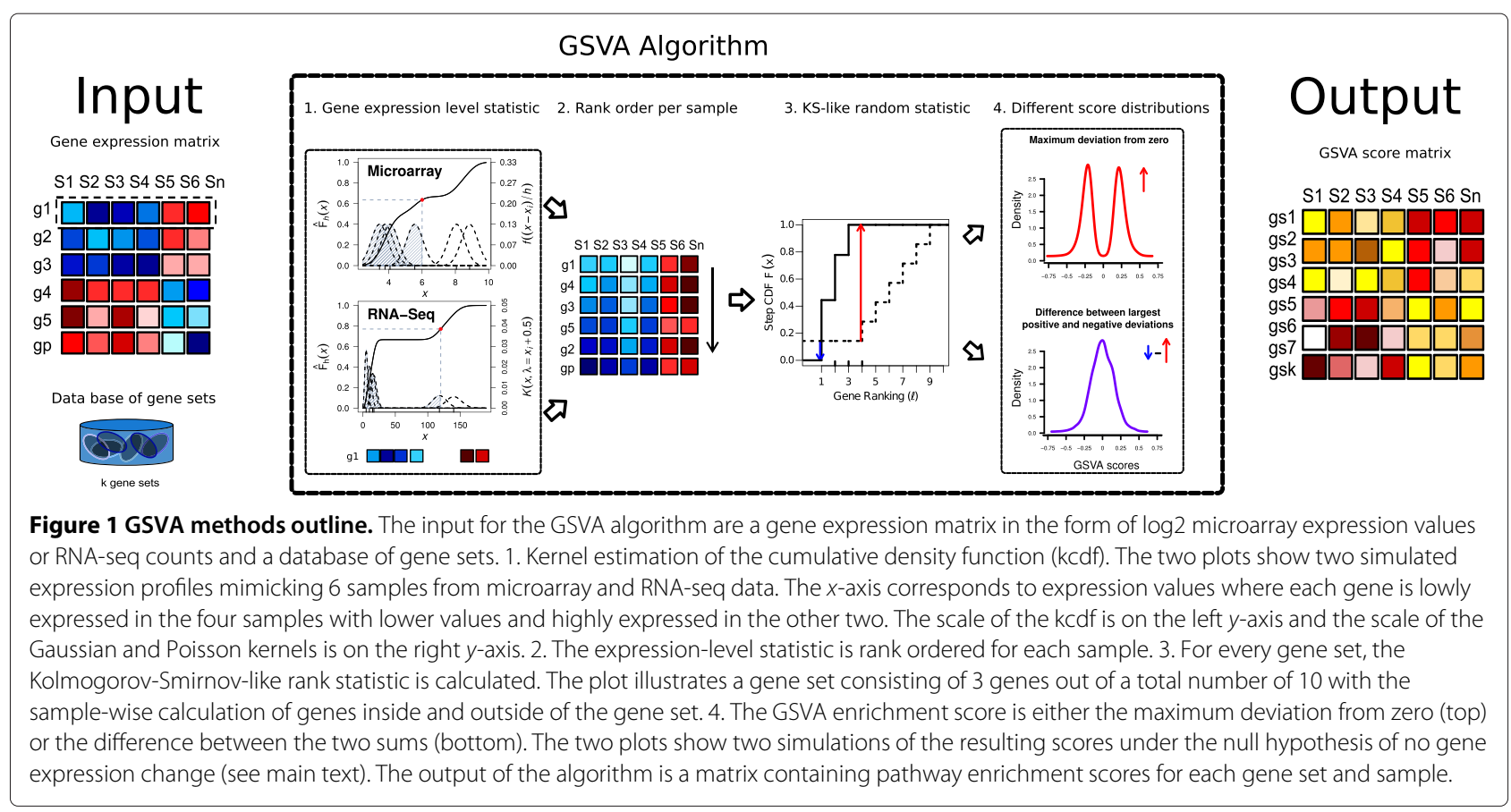


non-parametric kernel estimation of its cumulative density function is performed. In the case of microarray data, a Gaussian kernel ([25], pg. 148) is used:

$$
\hat{F}_{h_{i}}\left(x_{i j}\right)=\frac{1}{n} \sum_{k=1}^{n} \int_{-\infty}^{\frac{x_{i j}-x_{i k}}{h_{i}}} \frac{1}{\sqrt{2 \pi}} e^{-\frac{t^{2}}{2}} d t
$$

where $h_{i}$ is the gene-specific bandwidth parameter that controls the resolution of the kernel estimation, which is set to $h_{i}=s_{i} / 4$, where $s_{i}$ is the sample standard deviation of the $i$-th gene (Figure 1, step 1). In the case of RNA-seq data, a discrete Poisson kernel [26] is employed:

$$
\hat{F}_{r}\left(x_{i j}\right)=\frac{1}{n} \sum_{k=1}^{n} \sum_{y=0}^{x_{i j}} \frac{e^{-\left(x_{i k}+r\right)}\left(x_{i k}+r\right)^{y}}{y !},
$$

where $r=0.5$ in order to set the mode of the Poisson kernel at each $x_{i k}$, because the mode of a Poisson distribution with an integer mean $\lambda$ occurs at $\lambda$ and $\lambda-1$, and at the largest integer smaller than $\lambda$ when $\lambda$ is continuous.

Let $z_{i j}$ denote the previous expression-level statistic $\hat{F}_{h_{i}}\left(x_{i j}\right)$, or $\hat{F}_{r}\left(x_{i j}\right)$, depending on whether $x_{i j}$ are continuous microarray, or discrete count RNA-seq values, respectively. The following step condenses expressionlevel statistics into gene sets by calculating sample-wise enrichment scores. To reduce the influence of potential outliers, we first convert $z_{i j}$ to ranks $z_{(i) j}$ for each sample $j$ and normalize further $r_{i j}=\left|p / 2-z_{(i) j}\right|$ to make the ranks symmetric around zero (Figure 1, step 2). This is done to up-weight the two tails of the rank distribution when computing the final enrichment score.

We assess the enrichment score similar to the GSEA and ASSESS methods $[4,27]$ using the Kolmogorov-Smirnov (KS) like random walk statistic (Figure 1, step 3):

$$
v_{j k}(\ell)=\frac{\sum_{i=1}^{\ell}\left|r_{i j}\right|^{\tau} I\left(g_{(i)} \in \gamma_{k}\right)}{\sum_{i=1}^{p}\left|r_{i j}\right|^{\tau} I\left(g_{(i)} \in \gamma_{k}\right)}-\frac{\sum_{i=1}^{\ell} I\left(g_{(i)} \notin \gamma_{k}\right)}{p-\left|\gamma_{k}\right|},
$$

where $\tau$ is a parameter describing the weight of the tail in the random walk (default $\tau=1$ ), $\gamma_{k}$ is the $k$-th gene set, $I\left(g_{(i)} \in \gamma_{k}\right)$ is the indicator function on whether the $i$-th gene (the gene corresponding to the $i$-th ranked expression-level statistic) belongs to gene set $\gamma_{k},\left|\gamma_{k}\right|$ is the number of genes in the $k$-th gene set, and $p$ is the number of genes in the data set. Conceptually, Eq. 3 produces a distribution over the genes to assess if the genes in the gene set are more likely to be found at either tail of the rank distribution (see $[4,27]$ for a more detailed description).

We offer two approaches for turning the KS like random walk statistic into an enrichment statistic (ES) (also called GSVA score), the classical maximum deviation method
$[4,27,28]$ and a normalized ES. The first ES is the maximum deviation from zero of the random walk of the $j$-th sample with respect to the $k$-th gene set:

$$
E S_{j k}^{\max }=v_{j k}\left[\arg \max _{\ell=1, \ldots, p}\left|v_{j k}(\ell)\right|\right] .
$$

For each gene set $k$, this approach produces a distribution of enrichment scores that is bimodal (Figure 1, step 4, top panel, Additional file 1: Figure S1). This is an intrinsic property of the KS like random walk, which generates non-zero maximum deviations under the null distribution. In GSEA [4] it is also observed that the empirical null distribution obtained by permuting sample labels is bimodal and, for this reason, significance is determined independently using the positive and negative sides of the null distribution. In our case, we would like to provide a standard Gaussian distribution of enrichment scores under the null hypothesis of no change in pathway activity throughout the sample population. For this purpose we propose a second, alternative score that produces an ES distribution approximating this requirement (Figure 1, step 4, bottom panel, Additional file 1: Figure S1):

$E S_{j k}^{\mathrm{diff}}=\left|E S_{j k}^{+}\right|-\left|E S_{j k}^{-}\right|=\max _{\ell=1, \ldots, p}\left(0, v_{j k}(\ell)\right)-\min _{\ell=1, \ldots, p}\left(0, v_{j k}(\ell)\right)$,

where $E S_{j k}^{+}$and $E S_{j k}^{-}$are the largest positive and negative random walk deviations from zero, respectively, for sample $j$ and gene set $k$. This statistic may be compared to the Kuiper test statistic [29], which sums the maximum and minimum deviations to make the test statistic more sensitive in the tails. In contrast, our test statistic penalizes deviations that are large in both tails, and provides a "normalization" of the enrichment score by subtracting potential noise. There is a clear biological interpretation of this statistic, it emphasizes genes in pathways that are concordantly activated in one direction only, either over-expressed or under-expressed relative to the overall population. For pathways containing genes strongly acting in both directions, the deviations will cancel each other out and show little or no enrichment. Because this statistic is unimodal and approximately normal (as observed via simulation, see below), downstream analyses which may impose distributional assumptions on the data are thus possible. In certain cases, the characteristics of this statistic may be undesirable, especially if the relevant gene sets are not explicitly separated into "up" and "down" behavior (as the MSigDB provides for many gene sets). In such circumstances, the statistic defined by Eq. 4 should be used.

Figure 1, step 4 and Additional file 1: Figure S1 show a simple simulation where standard Gaussian deviates are independently sampled from $p=20,000$ genes and $n=30$ samples, thus mimicking a null distribution of 
no change in gene expression. One hundred gene sets are uniformly sampled at random from the $p$ genes with sizes ranging from 10 to 100 genes. Using these two inputs, we calculate the maximum deviation ES and the normalized ES. The resulting distributions are depicted in Figure 1, step 4 and Additional file 1: Figure S1.

Although the GSVA algorithm itself does not evaluate statistical significance for the enrichment of gene sets, significance with respect to a phenotype can be easily evaluated using conventional statistical models. Likewise, false discovery rates can be estimated by permuting the sample labels (Methods). We make no general prescription for thresholds of significance or false discovery, as these choices are highly context dependent and may vary according to each experiment. Examples of these techniques are provided in the following section.

\section{Results}

\section{Review of other methods}

Methods for gene set enrichment can be generally partitioned according to the criteria of supervised vs unsupervised, and population vs single sample assessments. Most GSE methods, such as GSEA [4], are supervised and population based, in that they compute an enrichment score per gene set to describe the entire data set, modeled on a phenotype (discrete, such as case-control, or continuous). The simplest of this genre is described by Tian et al. $[6,19]$, evaluated as the mean differential expression (e.g. case vs control) of a set of genes, compared to those genes not in the gene set. One of the major drawbacks of this method is that gene correlations are not taken into account, which might lead to an increased number of false-positive gene sets with respect to GSEA [30]. Many other supervised, population based approaches have also been described $[12,17,20,31-34]$.

A supervised, single sample based approach was introduced in the ASSESS method [27]. After dichotomizing the samples based on phenotypic classes, the ASSESS method computes density estimates for each gene/class followed by the evaluation of an enrichment score for each sample/gene set. This method is well-suited for assessing gene set variation across a dichotomous phenotype. GSVA also utilizes density estimates for evaluating sample-wise enrichment, but by omitting phenotypic information, it enables more general downstream analyses and therefore broader applications.

Three unsupervised, single sample enrichment methods have been developed, Pathway Level analysis of Gene Expression (PLAGE), single sample GSEA (ssGSEA) and the combined $\mathrm{z}$-score $[5,22,35]$. These methods compute an enrichment score for each gene set and individual sample. PLAGE standardizes each gene expression profile over the samples and then estimates the pathway activity profiles for each gene set as the coefficients of the first right-singular vector of the singular value decomposition of the gene set ([35], pg. 9). The combined z-score method [22] standardizes first, as PLAGE, each gene expression profile into $\mathrm{z}$-scores but the pathway activity profile is then obtained by combining the individual gene z-scores per sample ([22], Figure one). Both, PLAGE and the combined $\mathrm{z}$-score are parametric and assume that gene expression profiles are jointly normally distributed. The combined zscore additionally assumes that genes act independently within each gene set. The ssGSEA method from Barbie et al. [5] uses the difference in empirical cumulative distribution functions of gene expression ranks inside and outside the gene set to calculate an enrichment statistic per sample which is further normalized by the range of values taken throughout all gene sets and samples.

\section{Comparison of methods on simulated data}

GSVA is unsupervised and yields single sample enrichment scores. Therefore, we can directly compare the performance of GSVA to the combined z-score, single sample GSEA and PLAGE [5,22,35]. However, in contrast to the other methods, GSVA calculates first an expression statistic with the kernel estimation of the ECDF over the samples, which should help in protecting the method against systematic gene specific effects, such as probe effects, and therewith increase its sensitivity. To verify this hypothesis we have performed the following three simulation studies.

In the first study, we simulated microarray data from a linear additive model with sample and probe effects for $p=1,000$ genes and two groups of samples (see Methods). Using this model we have generated data sets of increasing sample size and defined two gene sets formed by 30 genes each, where one gene set is differentially expressed (DE) and the other is not. For the DE gene set we considered strong and weak signal-to-noise ratios and two different fractions of DE genes (50\% and 80\%) resulting in four different simulation scenarios. Using the simulated data from each scenario, we have calculated pathway activity profiles with the four sample-wise GSE methods (GSVA, ssGSEA, PLAGE and the combined $z$ score) and applied a $t$-test on the DE and non-DE gene sets between the two groups of samples. Using the DE gene set and a significance threshold of $\alpha=0.05$, we have estimated the statistical power of each method as function of the sample size. On the same data, but using the non-DE gene set, we have estimated the empirical type-I error rate at $\alpha=0.05$. The results of this simulation in Figure 2 show that GSVA attains higher statistical power than the other three methods in each of the four simulated scenarios while providing similar control of the type-I error rate.

In the second simulation study, we compared the accuracy of each GSE method to identify differential pathway activity by calling DE gene sets. For this, we used the 


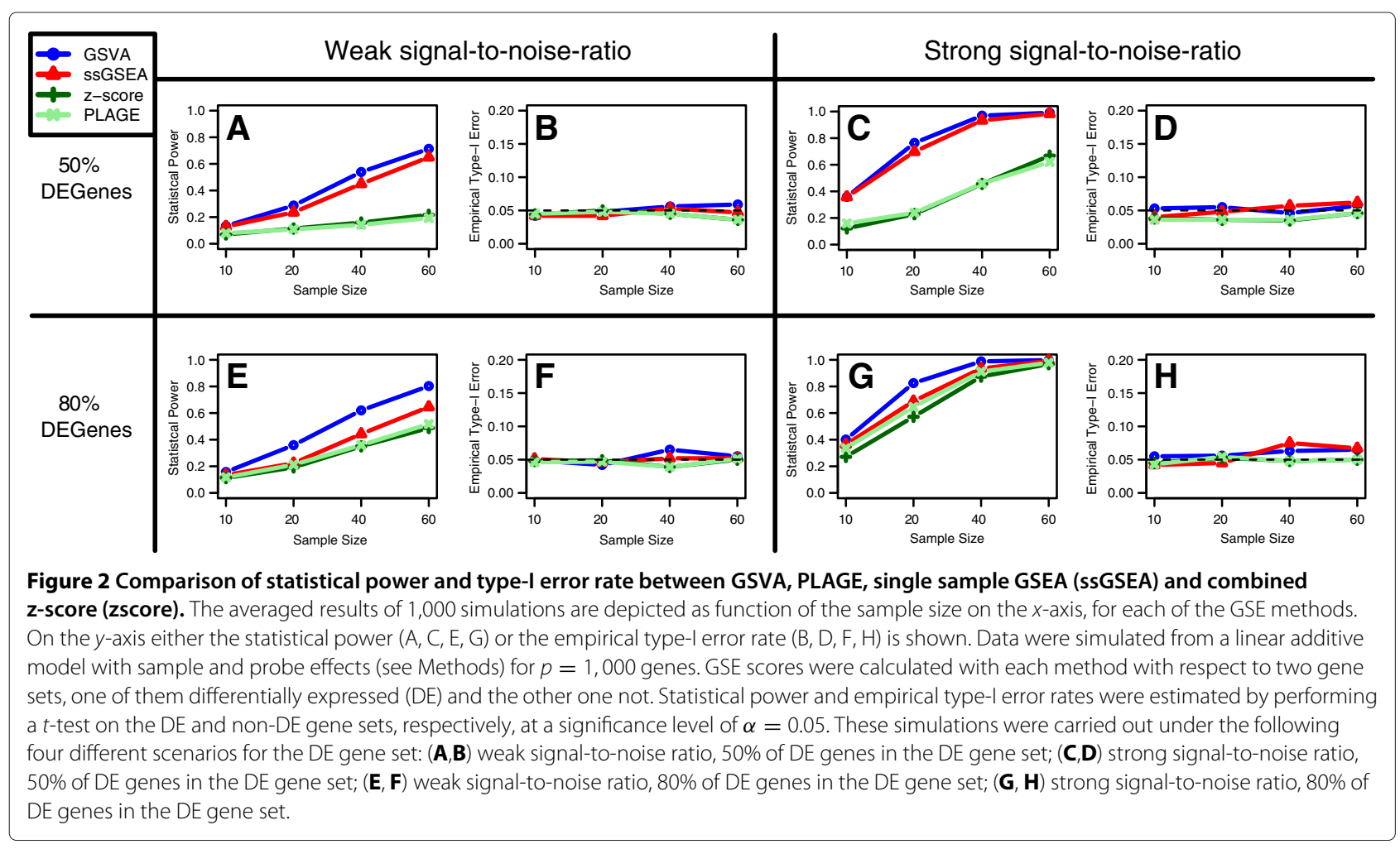

previously defined four simulation scenarios as well as the linear additive model with a fixed sample size of $n=60$ and $p=10,000$ genes to simulate data of more realistic dimensions. We set the first 2,000 genes as DE and simulated 1,000 gene sets of which we defined 500 as DE (see Methods). For each simulated gene expression data set, GSE scores were calculated and a two-sample $t$-test was employed to call DE gene sets at 5\% FDR. The performance of each GSE method was measured by the area under the ROC curve (AUC) across 100 independent simulations (see Methods). AUC values were calculated from the binary vector of DE calls to compare the ability of each method to identify DE gene sets at a genome-wide significance level. The results are shown in Figure 3. This figure shows that GSVA attains significantly higher mean AUC values than the other GSE methods $(P<0.05)$ in all but two of the twelve pairwise comparisons. This improvement in performance of GSVA over the other methods is also observed at a more stringent FDR cutoff of $1 \%$ (Additional file 1: Figure S2).

Finally, we carried out a third simulation study in the context of survival analysis. We used again the former linear additive model to simulate microarray data with $p=1,000$ genes and two groups of samples. This time, however, we performed a cross-validation study to assess predictive power using 50 gene sets, each consisting of 10 genes. One of the gene sets was set as DE between the two sample groups while the other 49 were not DE and formed by sampling uniformly at random among the other 990 genes. We used a fixed configuration on the magnitude of differential expression (strong) and on the fraction of DE genes in the DE gene set (50\%). In a similar way to the survival simulation by Bair and Tibshirani [36], we generated survival times and censoring status for each observation with different parameters for each group of samples (see Methods). This setting was generated twice to have independent training and test data sets.

GSE scores were calculated separately on the training and test data. A Cox proportional hazards model (Cox PHM) was fitted to each GSE score profile in the training data. The model with the lowest $\mathrm{p}$-value provided by the Wald test was used to predict risk on the test data. As baseline comparison, we also fitted a Cox PHM to each gene expression profile on the training data and selected the 10 genes, corresponding to the gene set size across all gene sets, with lowest $\mathrm{p}$-values given by the Wald test to also predict risk on the test data.

The performance of each gene set and gene-level model (using 10 genes) on the test data was assessed by the concordance index. This simulation was repeated 100 times and four entire runs were performed on increasing sample sizes $n=\{25,50,75,100\}$ of the simulated data. In Figure 4 the distribution of concordance index values is reported separately for each method and sample size. GSVA provides higher mean and median concordance index values than the other methods at every of the four 


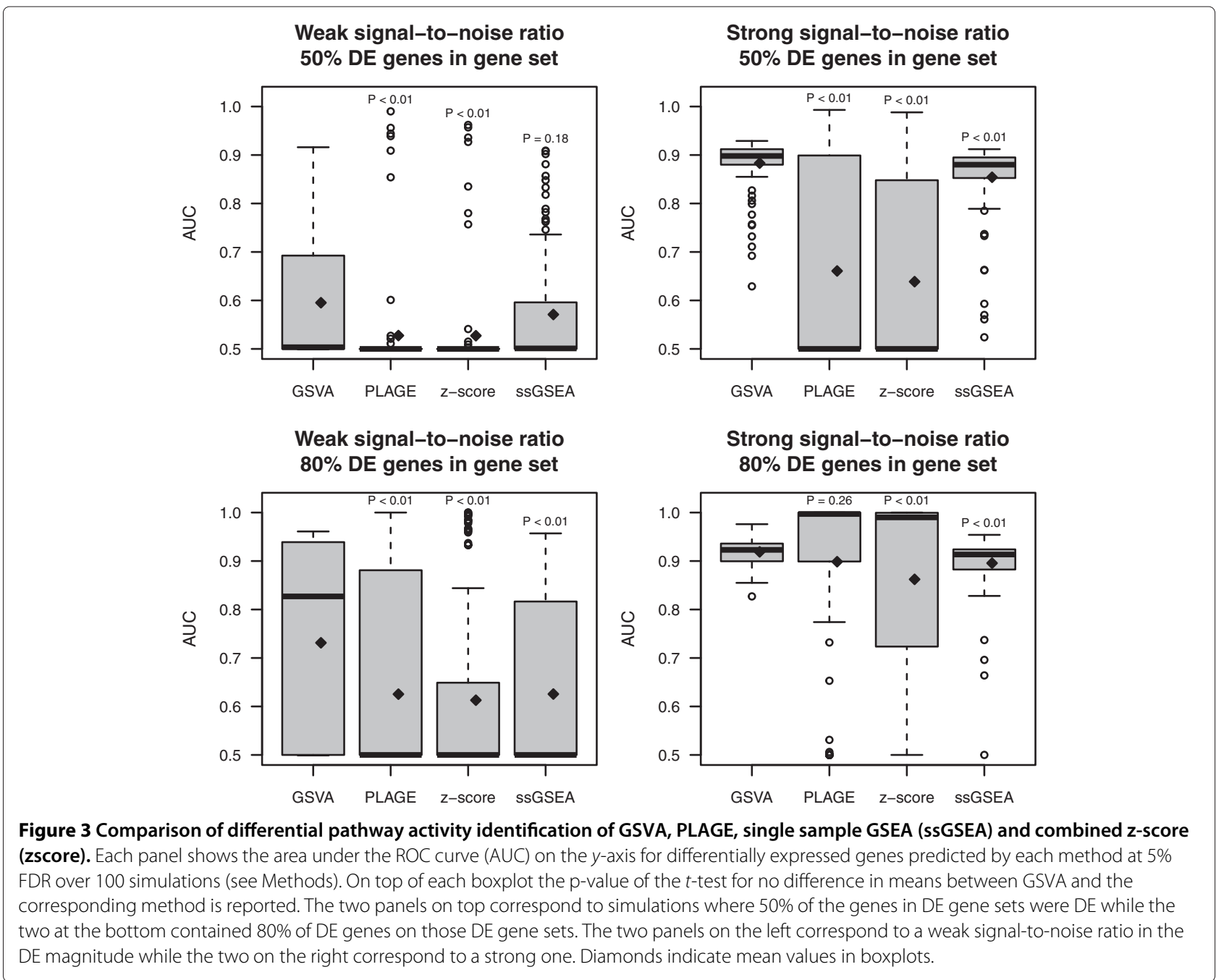

sample sizes and the difference in means is significant $(P<0.05)$ when $n \geq 50$.

\section{Lymphoblastic Leukemia: ALL vs MLL}

A canonical use of pathway-centric methods is the study of how pathway or gene set variation reveals the underlying biological structure with respect to a given phenotype. An example of this type of analysis was demonstrated in Verhaak, et al [28], where they showed how murinederived neuronal gene sets revealed a corresponding structure for glioblastoma subtypes in a large human cohort. To assess the higher power of GSVA to detect differentially expressed gene sets relevant to a phenotype of interest in real data, we have used a human leukemia data set. The data set consists of 37 different individuals with leukemia, of which 20 correspond to acute lymphoblastic leukemia (ALL) and 17 to mixed-lineage leukemia (MLL) [37]. We assessed the performance of the four samplewise GSE methods by evaluating their ability to produce a signature of the phenotype ALL vs MLL within different scenarios of magnitude of expression change.

We began by ranking all genes by fold change. Then, we partitioned this ranking into three equally sized fractions depicted in red, violet and blue in the volcano plot in Figure 5, panel A. We used each tercile of genes with increasing fold changes and bootstrap 10 samples from each class 1000 times. We applied the four GSE methods to the bootstrapped data together with the canonical Broad C2 collection of gene sets [4]. Subsequently, we performed differential expression analysis on the enrichment scores using limma [38]. From each ranking of adjusted p-values we selected the top 5 gene sets and used their enrichment scores to make a hierarchical clustering of the samples. We finally partitioned the samples into two groups using the two main branches of the hierarchy and calculated the adjusted rand index (ARI) [39] with respect to the corresponding sample label to assess the robustness of the clustering. 

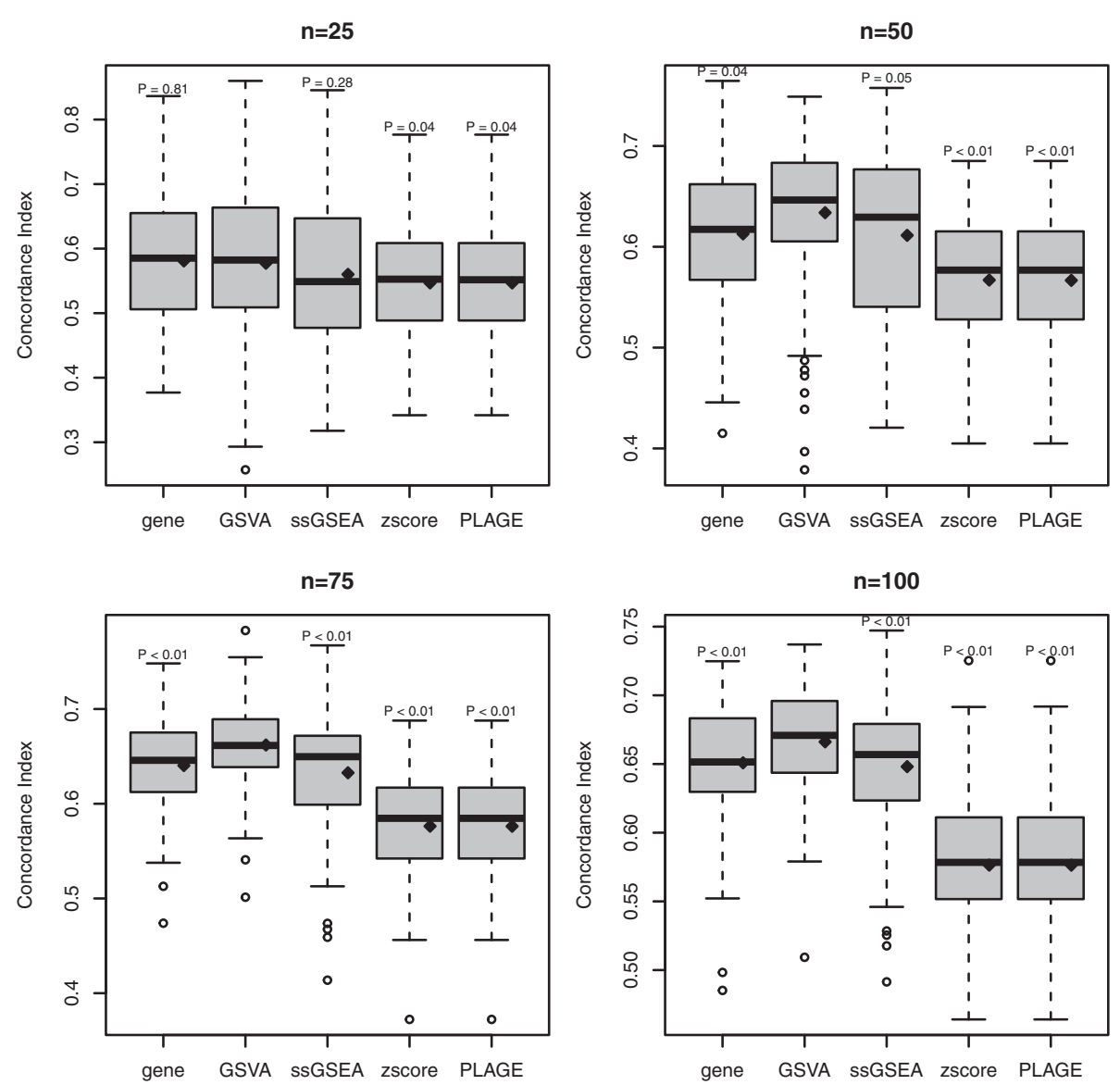

Figure 4 Comparison of the predictive power for survival analysis of gene-level, GSVA, PLAGE, single sample GSEA (sSGSEA) and combined z-score (zscore) on simulated data. Each panel corresponds to a different sample size of the simulated data. The $y$-axis shows the concordance index values of predicting survival risk on test data from 100 independent simulations. On top of each boxplot the p-value of the $t$-test for no difference in means between GSVA and the corresponding method is reported. The method gene refers to a simple gene-level survival model built from the top 10 genes with lowest p-values reported by the Wald test performed on the training data. Diamonds indicate mean values in boxplots.

As Figure 5 shows, ARI values depend on the tercile of fold change magnitude considered. Except in the case of the genes belonging to the tercile with largest fold changes (panel D), GSVA produced enrichment scores that led to significantly higher ARI values ( $t$-test for difference in means $\mathrm{p}$-value $<2 e-16$ ) than ssGSEA, PLAGE or the combined z-score approaches, demonstrating the larger power of GSVA to produce signatures capable of detecting subtle gene expression changes. Sample-wise enrichment scores easily enable extending this kind of analysis to a more complex phenotype with three or more sample groups. Such an example using adrenocortical carcinoma data can be found in Additional file 1: Figure S3 and Table S1.

\section{Survival analysis in ovarian carcinoma}

We next examined pathway models for predicting patient survival in ovarian serous cystadenocarcinoma (OV). We used a large gene expression experiment $(n=588)$ from
TCGA [40] to obtain pathway enrichment scores for each of the canonical gene sets (C2) in MSigDB, and compared the four GSE methods. We performed a five-fold crossvalidation and calculated GSE scores separately on each training and testing partition of the data with each of the four compared methods. We also considered the original expression data for a simple gene-level model. On each of the training data sets, we fitted a Cox PHM for each gene set, and each gene, in the gene-level model. Then, we selected those five gene-sets, or genes in the genelevel model, with the lowest p-value of the Wald test for no effect on survival. Using the selected gene-sets, we fitted again a Cox PHM on the training data and used it to predict risk on the training and test data sets of GSE scores. We repeated this for the gene-level model. Finally, we assessed the predictive performance of those models, each of them representing a different method, by calculating the concordance index of the predicted risk. As Figure 6 shows, except for the training data set using the 

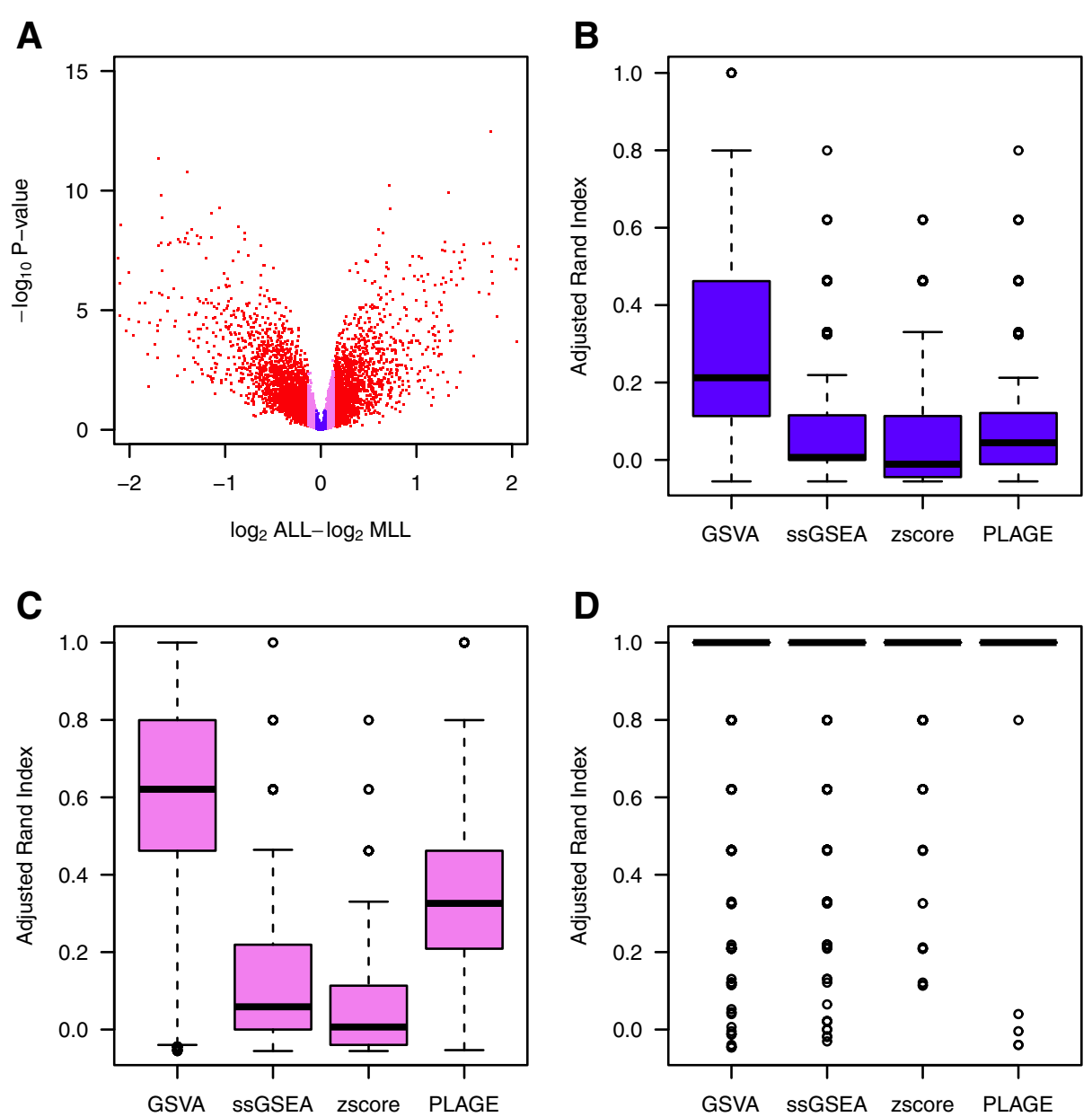

Figure 5 Comparison of differential pathway activity identification of GSVA, PLAGE, single sample GSEA (ssGSEA) and combined z-score (zscore) on a leukemia data set. (A) Volcano plot of gene expression changes in the Leukemia data set. Genes highlighted in red form the first tercile of largest absolute fold changes, violet indicates the second tercile and blue the third tercile. (B-D) Adjusted rand index (ARI) indicating the accuracy of classifying the two groups of samples by hierarchical clustering of the enrichment scores produced by each of the compared methods at the top- 5 differentially activated gene sets. The distribution of ARI values is formed by bootstrapping 1,000 times 10 samples from each sample group. Colors match the key given for genes in the volcano plot of (A) and show that, as expected, genes with larger fold changes lead to larger ARI values. However, when fold changes are small (B-C) and the underlying signature becomes extremely subtle, GSVA produces enrichment scores that lead to differentially activated gene sets which classify the two sample groups substantially better than using ssGSEA, zscore or PLAGE.

gene-level model, GSVA attains higher mean and median concordance index values than the other methods in both, training and testing data sets.

One of the main benefits of pathway-centric approaches is the interpretability they provide in understanding the mechanisms of disease. In Table 1, we list the top gene sets associated to survival as identified by GSVA (a complete list is available in Additional file 1: Table S2). False discovery rates (FDR) are re-estimated using a permutation based approach by randomly ordering the sample labels (patient survival times) 100 times, resulting in FDR estimates of 0.05 and 0.2 for $\mathrm{p}$-value thresholds of $10^{-4}$ and $5 \cdot 10^{-3}$, respectively. The first and second ranked gene sets suggest two important survival mechanisms:
DNA repair and modulation of the innate and adaptive immunity, respectively. Further inspection of the top significant gene sets $\left(P<10^{-3}\right)$ show that many of them are involved in wound and immune response. Interestingly, the 3rd and 13th ranked gene sets are derived from response signatures to tretinoin treatment, an alltrans retinoic acid drug that has been shown to suppress growth in ovarian cancer cell lines [41,42]. Finally, among the top 20 gene sets we note the presence of several EGF and RAS related pathways. While EGFR and RAS mutants are not commonly observed in ovarian cancer [43], activation of these well-studied oncogenes may still play an important role in progression and survival in ovarian cancer. 

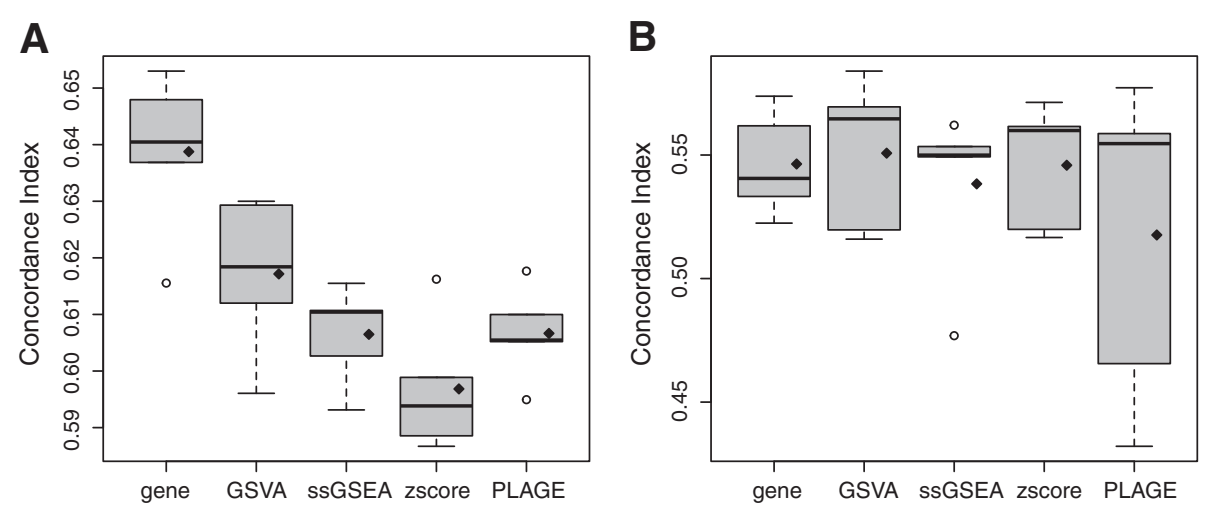

Figure 6 Survival analysis in a TCGA ovarian cancer data set. Predictive performance in the survival analysis of a TCGA ovarian cancer microarray data set of $n=588$ samples, measured by the concordance index obtained from a 5 -fold cross-validation from (A) the training data and (B) the test data. Diamonds indicate means in boxplots. Except in the training data using the gene-level model, GSVA provides higher mean and median concordance index values than the other compared methods in both training and testing cross-validated data sets.

\section{GSVA for RNA-seq data}

The application of high-throughput sequencing to interrogate RNA concentration in biological samples, popularly known as RNA-seq, is steadily becoming the technology of choice to profile gene expression [44]. The resulting sequence-based measurements take the form of discrete count data and yield a larger dynamic range and unbiased power than microarray technology to survey the cellular state of entire transcriptomes. The nature of these data, however, often requires specific statistical models and bioinformatic methods for their analysis, as in the case of differential expression analysis [45]. This is also the case of many GSE methods developed for microarray data which make distributional assumptions that preclude their direct application to RNA-seq count data $[1,46]$.

To our knowledge, no attempt has been made to condense gene-level RNA-seq expression profiles into gene sets to capture subtle changes in gene expression. GSE methods exist that either work with closed lists of differentially expressed genes (e.g. topGO [47], GOseq [48]), or rankings of some differential expression statistic, such as GSEA [4] and the mean-rank gene set enrichment method [49]. GOseq [48] is specifically designed to address gene length biases in lists of differentially expressed genes

Table 1 Top 5 pathways predictive of survival in ovarian cancer

\begin{tabular}{lc}
\hline Pathways & Cox P-value \\
\hline SIMBULAN_UV_RESPONSE_NORMAL_DN & $7.21 \times 10^{-6}$ \\
BIOCARTA_VIP_PATHWAY & $1.38 \times 10^{-5}$ \\
ZIRN_TRETINOIN_RESPONSE_WT1_UP & $3.38 \times 10^{-5}$ \\
DASU_IL6_SIGNALING_SCAR_UP & $3.46 \times 10^{-5}$ \\
WANG_HCP_PROSTATE_CANCER & $3.65 \times 10^{-5}$ \\
\hline
\end{tabular}

FDR evaluated as .05 with $p$-value threshold of $10^{-4}$. derived from RNA-seq data. But GOseq ignores genes that are not considered as differentially expressed and removes them from the analysis, hence ignoring genes with subtle changes. Also, rank-based methods ignore relative changes of genes in a pathway resulting in equal treatment of the genes, although they might have different fold changes [50]. Hence, these methods may be underpowered to detect subtle changes in pathway activity.

Here, we show how to apply GSVA to RNA-seq data. We provide pathway activity profiles analogous to the ones obtained from microarray data by using samples of lymphoblastoid cell lines (LCL) from HapMap individuals which have been profiled using both technologies [51,52]. Microarray and RNA-seq data were processed to obtain gene expression data matrices with matching gene and sample identifiers (Methods). The RNA-seq data consists of two tables of counts derived from reads obtained at two different sequencing centers, denoted by Argonne and Yale; see [52]. We calculated Spearman correlations for all genes and gene sets from both technologies. The resulting distributions of correlation values are shown in Figure 7, panels A and B, using the Argonne RNA-seq data (see Additional file 1: Figure S4 for analogous results for the Yale RNA-seq data). We show that GSVA enrichment scores correlate similarly to gene expression levels produced by both profiling technologies.

We also examined two gene sets containing genderspecific genes in detail: genes that escape $\mathrm{X}$-inactivation in female samples [53] and genes that are located on the male-specific region of the Y chrosomome [54]. Figure 7 illustrates that microarray and RNA-seq enrichment scores correlate very well in these gene sets, with $\rho=0.82$ for the male-specific gene set and $\rho=0.78$ for the female-specific gene set. Male and female samples show higher GSVA enrichment scores in their corresponding gene sets. This demonstrates the flexibility of 

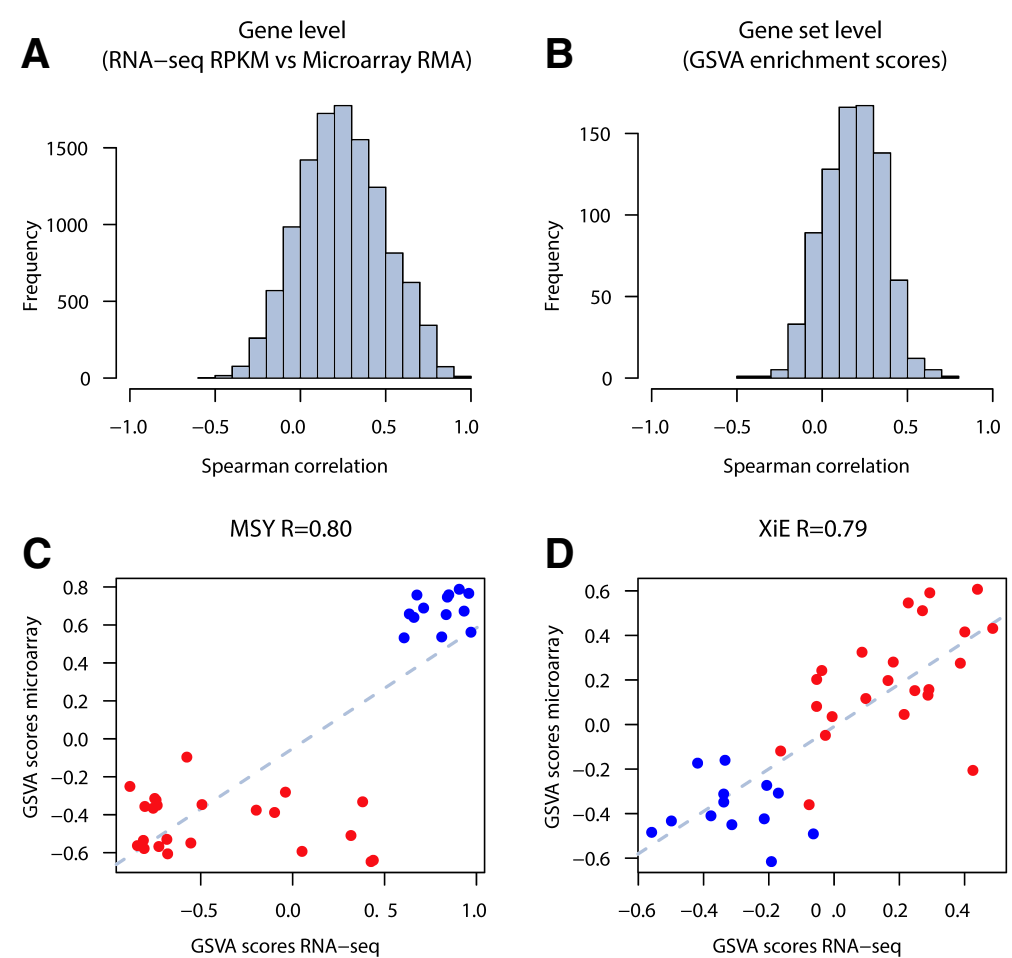

Figure 7 GSVA for RNA-seq (Argonne). A. Distribution of Spearman correlation values between gene expression profiles of RNA-seq and microarray data. B. Distribution of Spearman correlation values between GSVA enrichment scores of gene sets calculated from RNA-seq and microarray data. C and D. Comparison of GSVA enrichment scores obtained from microarray and RNA-seq data for two gene sets containing genes with sex-specific expression: MSY formed by genes of the male-specific region of the Y chromosome (male-specific), and XiE formed by genes that escape X-inactivation in females (female-specific). Red and blue points represent female and male samples, respectively. In both cases GSVA scores show very high correlation between the two profiling technologies where female samples show higher enrichment scores in the female-specific gene set and male samples show higher enrichment scores in the male-specific gene set.

GSVA to enable analogous unsupervised and single sample GSE analyses in data coming from both microarray and RNA-seq technologies.

\section{Methods}

\section{Simulations}

The simulation studies were carried out using the following linear additive model for mimicking normalized microarray data on $p$ genes and $n$ samples divided in two groups representing a case-control scenario:

$$
y_{i j}=\alpha_{i}+\beta_{j}+\epsilon_{i j}
$$

where $\alpha_{i} \sim \mathcal{N}(\mu=0, \sigma=1)$ is a gene-specific effect, such as a probe-effect, with $i=1, \ldots, p, \beta_{j} \sim \mathcal{N}\left(\mu_{j}, \sigma_{j}\right)$ is a sample-effect with $j=1,2$ and $e_{i j} \sim \mathcal{N}(\mu=0, \sigma=1)$ corresponds to random noise.

When assessing statistical power and type-I error in Figure 2, we set $p=1,000$ genes, out of which the first 30 were considered to form a DE gene set and the next 30 a non-DE gene set. We considered four different sample sizes $n=\{10,20,40,60\}$ and two varying conditions leading to four different simulation scenarios: the fraction of differentially expressed genes in the gene set $50 \%$ and $80 \%)$ and the signal-to-noise ratio expressed as the magnitude of the mean sample effect in DE genes for one of the sample groups (weak and strong signal-to-noise ratio). For non-DE genes $\mu_{1}=\mu_{2}=0$ with $\sigma_{1}=\sigma_{2}=1$ while for DE genes $\mu_{2}=0.5$ for the weak effect, $\mu_{2}=1$ for the strong effect and $\sigma_{2}=0.5$. Using the model in Eq. (6) with these parameters, we simulated 1,000 independent data sets. For each of the four GSE methods we obtained a GSE score matrix for two gene sets (DE and non-DE) by $n$ samples. On each GSE score matrix, we performed a twosample $t$-test on the two gene sets for a difference in mean between the two groups of samples $\left(H_{0}: \mu_{1}-\mu_{2}=0\right)$ at a significance level $\alpha=0.05$. The statistical power was then estimated as 1 minus the fraction of non-rejections of the DE gene set and the empirical type-I error was estimated as the fraction of rejections of the non-DE gene set, across the 1,000 simulations.

In the second simulation study we considered $p=$ 10,000 genes out of which 2,000 were set as DE and from which 1,000 gene sets were built, 500 of them being DE. DE genes and gene sets were simulated using the 
previously described parameters and simulation scenarios. Non-DE gene sets were simulated by sampling from the $p=10,000$ genes uniformly at random while DE gene sets were simulated by sampling among DE and non-DE genes in the proportions ( $50 \%$ or $80 \%$ of DE genes) defined by the corresponding scenario. For each scenario, we sampled the data this way 100 times and calculated GSE scores using the four GSE methods for every resulting data set. Using those GSE scores we performed a two-sample $t$-test for each gene set and called DE those meeting FDR cutoffs of $5 \%$ and $1 \%$. Performance was assessed by calculating ROC curves and AUC values using the R package ROCR [55].

The simulation study assessing the predictive power of GSE scores for survival in Figure 4 was performed using linear additive model in Eq. 6, where $\mu_{2}=1$ was fixed for DE genes in one of the sample groups. Survival times were generated for each sample group from two normal distributions $\mathcal{N}(\mu=6, \sigma=2)$ and $\mathcal{N}(\mu=10, \sigma=2)$. Censoring times were generated from a normal distribution $\mathcal{N}(\mu=10, \sigma=3)$. A sample was considered to be censored when the censoring time was smaller than the survival time.

\section{Data}

Data for differential expression analysis was obtained from the following sources: Leukemia [37] (www. broadinstitute.org) and Adrenocortical Carcinoma [56] (http://www.ncbi.nlm.nih.gov/geo; GSE10927). Data for the ovarian analysis was downloaded from TCGA on April 2011. At the time of analysis, 389 samples were available that had clinical data, gene expression (Affy U133A), and CNV (Affy SNP 6.0). In all cases, TCGA Level 3 data was used. Gene expression data was batch corrected using ComBat [57]. RNA-seq data corresponded to HapMap [58] lymphoblastoid cell lines (LCL) of Yoruba individuals [52] and the processed tables of counts were downloaded from http://eqtl.uchicago.edu/RNA_ Seq_data/results. Matching microarray samples form part of a larger study by Huang and co-workers [51] (http:// www.ncbi.nlm.nih.gov/geo; GSE7792).

\section{Microarray data processing}

Data analysis was performed using the $\mathrm{R}$ [59] and Bioconductor [60] software. We selected chips which passed quality control using affyPLM [61]. AffyPLM fits models on probe set level to identify chips of lower quality. Relative Log Expression (RLE) values (comparing probe expression on each array against the median expression across all arrays) and Normalized Unscaled Standard Errors (NUSE) (standard error estimates obtained for each gene and standardized across arrays) are calculated and cut-offs applied to remove low-quality samples.
Chips whose processing batch was confounded with the outcome of interest are not considered in the analysis. Each remaining Affymetrix chip was background adjusted, normalized and $\log 2$ transformed using the Robust Multi-array Average (RMA) algorithm [62].

Genes that are not expressed over the detection level of the microarray or whose expression values have a limited variability through the samples do not provide discriminatory power and may compromise the statistical power of subsequent analyses. For this reason, we removed 50\% of the genes with lower variability as measured by the interquartile range (IQR) across the samples except in the LCL microarray data.

\section{RNA-seq data processing}

The RNA-seq data from Pickrell et al. (2010) [52] were produced at two sequencing centers, Argonne and Yale, and preprocessed by the authors into two separate tables of counts of 41,466 Ensembl genes by 80 and 81 samples, respectively. We use these two tables of counts, and refer the reader for details on read mapping and summarization into gene-level counts to the methods of the publication [52]. Some of the samples (11 from Argonne and 12 from Yale) were prepared and sequenced twice within each sequencing center. In these cases we kept the sample of deeper coverage obtaining a final number of 69 samples on each table. We further filtered genes with low expression by discarding those with a mean of less than 0.5 counts per million calculated in $\log _{2}$ scale resulting in tables of counts with 17,607 genes (Argonne) and 17,843 genes (Yale) by 69 samples and we kept genes present in both tables $(17,324)$. Next, we normalized these two tables of counts adjusting for gene length and $\mathrm{G}+\mathrm{C}$ content using the Bioconductor package cqn [24]. The corresponding gene length and $\mathrm{G}+\mathrm{C}$ content information was extracted from data deposited at the same site from where the tables of counts were downloaded.

In order to proceed with the comparison of GSVA enrichment scores between microarray and RNA-seq data, we further filtered these two normalized tables of counts in order to match the genes and samples obtained after processing the LCL microarray data from Huang and co-workers [51]. This step required first to translate Ensembl gene identifiers into Entrez gene identifiers and second to match gene and sample identifiers between microarray and RNA-seq data. After these two steps we obtained the two final tables of counts analyzed in this paper of 11,508 Entrez genes by 36 samples from which 23 correspond to female and 13 to male individuals.

\section{Gene sets database}

In all experiments, we used the gene sets database from the Molecular Signature Database version 3 (MSigDB) C2 collection (curated pathways) [4] with 833 canonical 
pathways and 2392 chemical and gene perturbations, unless otherwise stated. After mapping genes from an experiment to the gene set database, we ignore all gene sets with fewer than 10 genes or more than 500 genes.

\section{FDR and multiple hypothesis correction}

In most experiments, we use a permutation approach to estimate an empirical FDR at a specified p-value threshold. In several cases we report multiple hypothesis correction based on the Benjamini-Hochberg (B.H.) approach [63] to obtain corrected p-values. In general, multiple hypothesis correction on gene sets is problematic, as many gene sets are highly overlapping and therefore not merely correlated, but essentially duplicated. Our use of B.H. is likely a conservative estimate of FDR and therefore used primarily as a demonstration of statistical power.

\section{Discussion}

The analyses conducted on simulated and real data demonstrate that GSVA outperforms competing methods for modeling pathway variation across samples in the context of identification of differential pathway activity and survival analysis. However, given the large number of GSE methods published and available to the bioinformatic community, GSVA may not be the optimal tool for every expression data set. We recommend GSVA as an intermediate universal tool, providing summaries of pathway activity for more open-ended biological analysis. For specific applications, highly specialized algorithms optimized for addressing domain specific problems may outperform GSVA. The user should also be aware that the non-parametric density estimation within the GSVA algorithm requires a sufficient number of observations which, according to our analysis of statistical power in Figure 2, should be larger than $n=10$.

Non-specific filtering of genes in high-throughput experiments has been shown to increase the statistical power to detect significant changes in gene expression levels [64] and this observation is likely to hold at gene set level. We have used a simple non-specific filtering strategy of a minimum and maximum cutoff on the size of a gene set after gene identifiers have been matched between gene expression data and gene sets. However, other strategies based on expected features of biologically relevant gene sets could potentially be more helpful. For instance, genes that are part of the same gene set or pathway are more likely to be expressed coordinately and are expected to exhibit some degree of correlation. Gene sets containing correlated genes are more coherent and provide a higher biological signal than incoherent, uncorrelated gene sets [65]. Hence, removing functionally incoherent pathways could constitute an appealing non-specific filtering strategy to improve detection power at gene set level.

\section{Conclusions}

We have presented a method for assaying the variation of gene set enrichment over a sample population. The method is freely available as a Bioconductor package for $\mathrm{R}$ under the name GSVA at http://www.bioconductor.org. The increasing availability of large data sets with multiple assays and complex phenotypes has motivated our work because the study of these data sets within the context of pathways will be critical to their understanding. The GSVA method is both non-parametric and unsupervised, and bypasses the conventional approach of explicitly modeling phenotypes within the enrichment scoring algorithm. We have also shown how GSVA can be easily adapted to the analysis of RNA-seq data producing results analogous to its microarray counterpart. In the Additional file 1, two other examples of GSVA applications can be found including differential pathway analysis in a multiclass adrenocortical carcinoma data set (Additional file 1: Figure S3 and Table S1), and correlation analysis of pathways and copy-number alterations in ovarian carcinoma (Additional file 1: Figure S5).

For future directions, we believe GSVA may be used in genetical genomics strategies analogous to eQTL mapping to study, what we might call, pathway-QTL to identify DNA polymorphisms that impact pathway activity [66]. This could be extended further to support causal inference [67], where pathways replace genes in modeling the causal chain of genotype $\rightarrow$ gene expression $\rightarrow$ phenotype.

\section{Availability and requirements}

- Project name: GSVA

- Project home page: http://www.bioconductor.org/ packages/release/bioc/html/GSVA.html

- Operating system(s): Platform independent

- Programming language: $\mathrm{R}, \mathrm{C}$

- Other requirements: $R(>=2.15 .0)$, the $R$ package methods, and the Bioconductor package GSEABase $(>=1.18 .0)$

- License: GPL $(>=2)$

- Any restrictions to use by non-academics: no restrictions

\section{Additional file}

Additional file 1: Supplementary material including figures $\mathrm{S} 1$ to $\mathrm{S} 5$ and tables S1 and S2.

Competing interests

The authors declare no conflict of interest.

\section{Authors' contributions}

JG conceived and designed the GSVA algorithm. JG and RC implemented the software. SH and JG conceived and designed the applications of GSVA. SH, RC and JG analyzed the data and wrote the paper. All authors read and approved the final manuscript. 


\section{Acknowledgements}

We thank the following individuals for their helpful comments: Ingo Vogt, Alba Jene, Gunes Gundem, Sonja Althammer and Josh Millstein. S.H. and R.C. acknowledge support from an ISCIII COMBIOMED grant [RD07/0067/0001] and a Spanish MINECO grant [TIN2011-22826]. J.G. is supported in part by the National Cancer Institute Integrative Cancer Biology Program, grant U54CA149237.

\section{Author details}

${ }^{1}$ Research Program on Biomedical Informatics (GRIB), Hospital del Mar Medical Research Institute (IMIM), Barcelona, Catalonia, Spain. ${ }^{2}$ Department of Experimental and Health Sciences, Universitat Pompeu Fabra, Barcelona, Catalonia, Spain. ${ }^{3}$ Sage Bionetworks, 1100 Fairview Ave N., Seattle, Washington, 98109 USA.

Received: 22 May 2012 Accepted: 21 December 2012

Published: 16 January 2013

\section{References}

1. Goeman JJ, Geer SAvd, Kort Fd, Houwelingen HCV: A global test for groups of genes: testing association with a clinical outcome. Bioinformatics 2004, 20:93-99. [http://bioinformatics.oxfordjournals.org/ content/20/1/93.abstract]

2. Mootha VK, Lindgren CM, Eriksson KF, Subramanian A, Sihag S, Lehar J, Puigserver P, Carlsson E, Ridderstråle M, Laurila E, Houstis N, Daly MJ, Patterson N, Mesirov JP, Golub TR, Tamayo P, Spiegelman B, Lander ES, Hirschhorn JN, Altshuler D, Groop LC: PGC-1alpha-responsive genes involved in oxidative phosphorylation are coordinately downregulated in human diabetes. Nature Genet 2003, 34(3):267-273. [http://www.ncbi.nlm.nih.gov/pubmed/12808457]

3. Sweet-Cordero A, Mukherjee S, Subramanian A, You H, Roix J J, Ladd-Acosta C, Mesirov J, Golub TR, Jacks T: An oncogenic KRAS2 expression signature identified by cross-species gene-expression analysis. Nature Gen 2005, 37:48-55. [http://www.ncbi.nlm.nih.gov/ pubmed/15608639]

4. Subramanian A, Tamayo P, Mootha VK, Mukherjee S, Ebert BL, Gillette MA, Paulovich A, Pomeroy SL, Golub TR, Lander ES, Mesirov JP: Gene set enrichment analysis: A knowledge-based approach for interpreting genome-wide expression profiles. Proc Natl Acad Sci USA 2005, 102(43):15545-15550. [http://www.pnas.org/content/102/43/15545. abstract]

5. Barbie DA, Tamayo P, Boehm JS, Kim SY, Moody SE, Dunn IF, Schinzel AC, Sandy P, Meylan E, Scholl C, Fröhling S, Chan EM, Sos ML, Michel K, Mermel C, Silver SJ, Weir BA, Reiling JH, Sheng Q, Gupta PB, Wadlow RC, Le H, Hoersch S, Wittner BS, Ramaswamy S, Livingston DM, Sabatini DM, Meyerson M, Thomas RK, Lander ES, Mesirov JP, Root DE, Gilliland DG, Jacks T, Hahn WC: Systematic RNA interference reveals that oncogenic KRAS-driven cancers require TBK1. Nature 2009, 462(7269):108-112. [http://www.nature.com/nature/journal/v462/ n7269/abs/nature08460.html]

6. Tian L, Greenberg SA, Kong SW, Altschuler J, Kohane IS, Park PJ: Discovering statistically significant pathways in expression profiling studies. Proc Natl Acad Sci USA 2005, 102(38):13544-13549. [http://www. pnas.org/content/102/38/13544]

7. Barry WT, Nobel AB, Wright FA: Significance analysis of functional categories in gene expression studies: a structured permutation approach. Bioinformatics 2005, 21(9):1943-1949. [http://www.ncbi.nlm. nih.gov/pubmed/15647293]

8. Efron B, Tibshirani R: On testing the significance of sets of genes. Ann App/ Stat 2006, 1(1):107-129. [http://arxiv.org/abs/math/0610667]

9. Dørum G, Snipen L, Solheim M, Sæbø S: Rotation testing in gene set enrichment analysis for small direct comparison experiments. Stat Apps Gen Mol Bio 2009, 8: [http://www.bepress.com/sagmb/vol8/iss1/ art34]

10. Irizarry RA, Wang C, Zhou Y, Speed TP: Gene set enrichment analysis made simple. Stat Methods Med Res 2009, 18(6):565-575. [http://smm. sagepub.com/content/18/6/565.abstract]

11. Jiang Z, Gentleman R: Extensions to gene set enrichment. Bioinformatics 2007, 23(3):306-313. [http://bioinformatics.oxfordjournals org/content/23/3/306.abstract]
12. Wu D, Lim E, Vaillant F, Asselin-Labat ML, Visvader JE, Smyth GK: ROAST: rotation gene set tests for complex microarray experiments. Bioinformatics (Oxford, England) 2010, 26(17):2176-2182. [http://www. ncbi.nlm.nih.gov/pubmed/20610611]. [PMID: 20610611]

13. Lamb J, Ramaswamy S, Ford HL, Contreras B, Martinez RV, Kittrell FS, Zahnow CA, Patterson N, Golub TR, Ewen ME: A mechanism of cyclin D1 action encoded in the patterns of gene expression in human cancer. Cell 2003, 114(3):323-334. [http://www.cell.com/abstract/S00928674(03)00570-1]

14. Shepard JL, Amatruda JF, Stern HM, Subramanian A, Finkelstein D, Ziai J, Finley KR, Pfaff KL, Hersey C, Zhou Y, Barut B, Freedman M, Lee C, Spitsbergen J, Neuberg D, Weber G, Golub TR, Glickman JN, Kutok JL, Aster JC, Zon LI: A zebrafish bmyb mutation causes genome instability and increased cancer susceptibility. Proc Natl Acad Sci USA 2005, 102(37):13194-13199. [http://www.pnas.org/content/102/37/ 13194.abstract]

15. Segrè AV, Groop L, Mootha VK, Daly MJ, Altshuler D, Consortium D, investigators M: Common inherited variation in Mitochondrial genes is not enriched for associations with Type 2 diabetes or related glycemic Traits. PLoS Genet 2010, 6(8):e1001058. [http://dx.doi.org/10. 1371/journal.pgen.1001058]

16. Pece S, Tosoni D, Confalonieri S, Mazzarol G, Vecchi M, Ronzoni S, Bernard L, Viale G, Pelicci PG, Fiore PPD: Biological and molecular heterogeneity of breast cancers correlates with their cancer stem cell content. Cell 2010, 140:62-73. [http://www.sciencedirect.com/science/article/B6WSN4Y3TDSF-D/2/9fd74fc1accc422d7a6e6d935b45975c]

17. Hung JH, Yang TH, Hu Z, Weng Z, DeLisi C: Gene set enrichment analysis: performance evaluation and usage guidelines. Brief Bioinformatics 2012, 13(3):281-291. [http://www.ncbi.nlm.nih.gov/ pubmed/21900207]. [PMID: 21900207]

18. Goeman JJ, Bühlmann P: Analyzing gene expression data in terms of gene sets: methodological issues. Bioinformatics (Oxford, England) 2007, 23(8):980-987. [http://www.ncbi.nlm.nih.gov/pubmed/17303618]. [PMID: 17303618]

19. Kim SY, Volsky DJ: PAGE: Parametric analysis of gene set enrichment. BMC Bioinformatics 2005, 6:144. [PMID: 15941488 PMCID: 1183189]

20. Tenenbaum JD, Walker MG, Utz PJ, Butte AJ: Expression-based Pathway Signature Analysis (EPSA): Mining publicly available microarray data for insight into human disease. BMC Med Genomics 2008, 1:51 [http://www.biomedcentral.com/1755-8794/1/51]

21. Creighton CJ: Multiple oncogenic pathway signatures show coordinate expression patterns in human prostate tumors. PLOS One 2008, 3(3):e1816. [http://dx.doi.org/10.1371/journal.pone.0001816]

22. Lee E, Chuang HY, Kim JW, Ideker T, Lee D: Inferring pathway activity toward precise disease classification. PLoS Comput Biol 2008, 4(11):e1000217. [http://dx.doi.org/10.1371/journal.pcbi.1000217]

23. Zilliox MJ, Irizarry RA: A gene expression bar code for microarray data. Nat Meth 2007, 4(11):911-913. [http://dx.doi.org/10.1038/nmeth1102]

24. Hansen KD, Irizarry RA, Wu Z: Removing technical variability in RNA-seq data using conditional quantile normalization. Biostatistics 2012. [http://biostatistics.oxfordjournals.org/content/early/2012/01/24/ biostatistics.kxr054.abstract]

25. Silverman BW: Density Estimation for Statistics and Data Analysis. London: Chapman and Hall; 1986. [http://www.crcpress.com/product/isbn/ 9780412246203]. [ISBN 9780412246203]

26. Canale A, Dunson DB: Bayesian kernel mixtures for counts. J Am Stat Assoc 2011, 106(496): 1528-1539.

27. Edelman E, Porrello A, Guinney J, Balakumaran B, Bild A, Febbo PG, Mukherjee $S$ : Analysis of sample set enrichment scores: assaying the enrichment of sets of genes for individual samples in genome-wide expression profiles. Bioinformatics 2006, 22(14):e108-e116. [http:// www.ncbi.nlm.nih.gov/pubmed/16873460]

28. Verhaak RGW, Hoadley KA, Purdom E, Wang V, Qi Y, Wilkerson MD, Miller CR, Ding L, Golub T, Mesirov JP, Alexe G, Lawrence M, O'Kelly M, Tamayo P, Weir BA, Gabriel S, Winckler W, Gupta S, Jakkula L, Feiler HS, Hodgson JG, James CD, Sarkaria JN, Brennan C, Kahn A, Spellman PT, Wilson RK, Speed TP, Gray JW, Meyerson M, Getz G, Perou CM, Hayes DN: Integrated genomic analysis identifies clinically relevant subtypes of glioblastoma characterized by abnormalities in PDGFRA, IDH1, EGFR, and NF1. Cancer Cell 2010, 17:98-110. [http://www.ncbi.nlm.nih. gov/pubmed/20129251] 
29. Pearson E: Comparison of tests for randomness of points on a line. Biometrika 1963, 50:315-325.

30. Tamayo P, Steinhardt G, Liberzon A, Mesirov JP: Gene set enrichment analysis made right. arXiv:1110.4128 2011. [http://arxiv.org/abs/1110. 4128]

31. Khatri $P$, Drăghici S: Ontological analysis of gene expression data: current tools, limitations, and open problems. Bioinformatics 2005, 21(18):3587-3595. [http://bioinformatics.oxfordjournals.org/content/21/ 18/3587]

32. Nam D, Kim SY: Gene-set approach for expression pattern analysis. Brief Bioinformatics 2008, 9(3):189-197. [http://bib.oxfordjournals.org/ content/9/3/189]

33. Huang DW, Sherman BT, Lempicki RA: Bioinformatics enrichment tools: paths toward the comprehensive functional analysis of large gene lists. Nucleic Acids Res 2009, 37:1-13. [http://www.ncbi.nlm.nih. gov/pmc/articles/PMC2615629/]. [PMID: 19033363 PMCID: PMC2615629]

34. Jung $\mathrm{K}$, Becker $\mathrm{B}$, Brunner $\mathrm{E}$, Beißbarth $\mathrm{T}$ : Comparison of global tests for functional gene sets in two-group designs and selection of potentially effect-causing genes. Bioinformatics 2011

27(10):1377-1383. [http://bioinformatics.oxfordjournals.org/content/27/ 10/1377]

35. Tomfohr J, Lu J, Kepler TB: Pathway level analysis of gene expression using singular value decomposition. BMC Bioinformatics 2005, 6:225. [http://www.ncbi.nlm.nih.gov/pmc/articles/PMC1261155/]. [PMID: 16156896 PMCID: PMC1261155]

36. Bair E, Tibshirani R: Semi-supervised methods to predict patient survival from gene expression data. PLoS Bio/ 2004, 2(4). [http://Www. ncbi.nlm.nih.gov/pmc/articles/PMC387275/]. [PMID: 15094809 PMCID: PMC387275]

37. Armstrong SA, Staunton JE, Silverman LB, Pieters R, Boer MLd, Minden MD, Sallan SE, Lander ES, Golub TR, Korsmeyer SJ: MLL translocations specify a distinct gene expression profile that distinguishes a unique leukemia. Nature Gen 2002, 30:41-47. [http://www.ncbi.nlm.nih. gov/pubmed/11731795]

38. Smyth GK: Linear models and empirical Bayes Methods for assessing differential expression in microarray experiments. Stat App/ Gen Mol Biol 2004, 3: [http://www.bepress.com/sagmb/vol3/iss1/art3]

39. Hubert L, Arabie P: Comparing partitions. J Classif 1985, 2:193-218. [http://www.springerlink.com/content/x64124718341j1j0/abstract/]

40. Network TCGAR: Integrated genomic analyses of ovarian carcinoma. Nature 2011, 474(7353):609-615. [http://www.ncbi.nlm.nih.gov/ pubmed/21720365]. [PMID: 21720365]

41. Soprano KJ, Purev E, Vuocolo S, Soprano DR: Rb2/p130 and protein phosphatase $2 \mathrm{~A}$ : key mediators of ovarian carcinoma cell growth suppression by all-trans retinoic acid. Oncogene 2006, 25(38):5315-5325. [http://www.ncbi.nlm.nih.gov/pubmed/16936753]. [PMID: 16936753]

42. Um SJ, Lee SY, Kim EJ, Han HS, Koh YM, Hong KJ, Sin HS, Park JS: Antiproliferative mechanism of retinoid derivatives in ovarian cancer cells. Cancer Letters 2001, 174(2):127-134. [http://www. sciencedirect.com/science/article/pii/S0304383501006978]

43. Forbes SA, Bindal N, Bamford S, Cole C, Kok CY, Beare D, Jia M, Shepherd R, Leung K, Menzies A, Teague JW, Campbell PJ, Stratton MR, Futreal PA: COSMIC: mining complete cancer genomes in the Catalogue of Somatic Mutations in Cancer. Nucleic Acids Res 2010, 39(Database):D945-D950. [http://nar.oxfordjournals.org/content/39/ suppl_1/D945.long]

44. Mortazavi A, Williams BA, McCue K, Schaeffer L, Wold B: Mapping and quantifying mammalian transcriptomes by RNA-Seq. Nat Meth 2008 , 5(7):621-628. [http://dx.doi.org/10.1038/nmeth.1226]

45. Robinson MD, McCarthy DJ, Smyth GK: edgeR: a Bioconductor package for differential expression analysis of digital gene expression data. Bioinformatics 2010, 26:139-140. [http://bioinformatics.oxfordjournals. org/content/26/1/139.short]

46. Wu D, Lim E, Vaillant F, Asselin-Labat ML, Visvader JE, Smyth GK: ROAST: rotation gene set tests for complex microarray experiments. Bioinformatics (Oxford, England) 2010, 26(17):2176-2182. [http://www. ncbi.nlm.nih.gov/pubmed/20610611]. [PMID: 20610611]

47. Alexa A, Rahnenführer J, Lengauer T: Improved scoring of functional groups from gene expression data by decorrelating GO graph structure. Bioinformatics 2006, 22(13):1600-1607. [http://bioinformatics. oxfordjournals.org/content/22/13/1600.abstract]

48. Young MD, Wakefield MJ, Smyth GK, Oshlack A: Gene ontology analysis for RNA-seq: accounting for selection bias. Genome Bio/ 2010, 11(2):R14. [http://www.ncbi.nlm.nih.gov/pubmed/20132535]. [PMID: 20132535]

49. Michaud J, Simpson KM, Escher R, Buchet-Poyau K, Beissbarth T, Carmichael C, Ritchie ME, Schütz F, Cannon P, Liu M, Shen X, Ito Y, Raskind WH, Horwitz MS, Osato M, Turner DR, Speed TP, Kavallaris M, Smyth GK, Scott HS: Integrative analysis of RUNX1 downstream pathways and target genes. BMC Genomics 2008, 9:363. [http://www.biomedcentral. com/1471-2164/9/363/abstract]

50. Khatri $P$, Sirota $M$, Butte AJ: Ten years of pathway analysis: current approaches and outstanding challenges. PLoS Comput Bio/ 2012, 8(2):e1002375. [http://dx.doi.org/10.1371/journal.pcbi.1002375]

51. Huang RS, Duan S, Bleibel WK, Kistner EO, Zhang W, Clark TA, Chen TX, Schweitzer AC, Blume JE, Cox NJ, Dolan ME: A genome-wide approach to identify genetic variants that contribute to etoposide-induced cytotoxicity. Proc Natl Acad Sci USA 2007, 104(23):9758-9763. [http:// www.ncbi.nlm.nih.gov/pubmed/17537913]. [PMID: 17537913]

52. Pickrell JK, Marioni JC, Pai AA, Degner JF, Engelhardt BE, Nkadori E, Veyrieras JB, Stephens M, Gilad Y, Pritchard JK: Understanding mechanisms underlying human gene expression variation with RNA sequencing. Nature 2010, 464(7289):768-772. [http://dx.doi.org/10. 1038/nature08872]

53. Carrel L, Willard HF: X-inactivation profile reveals extensive variability in X-linked gene expression in females. Nature 2005, 434(7031):400-404. [http://www.ncbi.n/m.nih.gov/pubmed/15772666]. [PMID: 15772666]

54. Skaletsky H, Kuroda-Kawaguchi T, Minx PJ, Cordum HS, Hillier L, Brown LG, Repping S, Pyntikova T, Ali J, Bieri T, Chinwalla A, Delehaunty A, Delehaunty K, Du H, Fewell G, Fulton L, Fulton R, Graves T, Hou SF, Latrielle P, Leonard S, Mardis E, Maupin R, McPherson J, Miner T, Nash W, Nguyen C, Ozersky P, Pepin K, Rock S, Rohlfing T, Scott K, Schultz B, Strong C, Tin-Wollam A, Yang SP, Waterston RH, Wilson RK, Rozen S, Page DC: The male-specific region of the human $Y$ chromosome is a mosaic of discrete sequence classes. Nature 2003, 423(6942):825-837. [http://dx. doi.org/10.1038/nature01722]

55. Sing T, Sander O, Beerenwinkel N, Lengauer T: ROCR: visualizing classifier performance in R. Bioinformatics 2005, 21(20):3940-3941.

56. Giordano TJ, Kuick R, Else T, Gauger PG, Vinco M, Bauersfeld J, Sanders D, Thomas DG, Doherty G, Hammer G: Molecular classification and prognostication of adrenocortical tumors by transcriptome profiling. Clin Cancer Res: Official J Am Assoc Cancer Res 2009, 15(2):668-676. [http://www.ncbi.nlm.nih.gov/pubmed/19147773]. [PMID: 19147773]

57. Johnson WE, Li C, Rabinovic A: Adjusting batch effects in microarray expression data using empirical Bayes methods. Biostatistics 2007, 8:118-127.

58. Frazer KA, Ballinger DG, Cox DR, Hinds DA, Stuve LL, Gibbs RA, Belmont JW, Boudreau A, Hardenbol P, Leal SM, Pasternak S, Wheeler DA, Willis TD, Yu F, Yang H, Zeng C, Gao Y, Hu H, Hu W, Li C, Lin W, Liu S, Pan H, Tang X, Wang J, Wang W, Yu J, Zhang B, Zhang Q, Zhao H, et al.: A second generation human haplotype map of over 3.1 million SNPs. Nature 2007, 449(7164):851-861. [http://www.nature.com/nature/journal/v449/ n7164/abs/nature06258.html]

59. Team RDC: R: A Language and Environment for Statistical Computing. Vienna: R Foundation for Statistical Computing; 2010. [http://www.Rproject.org]. [ISBN 3-900051-07-0]

60. Gentleman RC, Carey VJ, Bates DM, Bolstad B, Dettling M, Dudoit S, Ellis B, Gautier L, Ge Y, Gentry J, Hornik K, Hothorn T, Huber W, lacus S, Irizarry R, Leisch F, Li C, Maechler M, Rossini AJ, Sawitzki G, Smith C, Smyth G, Tierney L, Yang JYH, Zhang J: Bioconductor: open software development for computational biology and bioinformatics. Genome Biol 2004, 5(10):R80. [http://www.ncbi.nlm.nih.gov/pubmed/ 15461798]. [PMID: 15461798]

61. Bolstad BM: Low-level analysis of high-density oligonucleotide array data: background, normalization and summarization. PhD thesis, University of Waikato 2004. [http://bmbolstad.com/Dissertation/ Bolstad_2004_Dissertation.pdf] 
62. Irizarry RA, Bolstad BM, Collin F, Cope LM, Hobbs B, Speed TP: Summaries of Affymetrix GeneChip probe level data. Nucleic Acids Res 2003, 31(4):e15.

63. Benjamini $Y$, Hochberg $Y$ : Controlling the false discovery rate: a practical and powerful approach to multiple testing. J R Stat Soc 1995, 57:289-300.

64. Bourgon R, Gentleman R, Huber W: Independent filtering increases detection power for high-throughput experiments. Proc Natl Acad Sci 2010, 107(21):9546-9551. [http://www.pnas.org/content/107/21/9546]

65. Levine DM, Haynor DR, Castle JC, Stepaniants SB, Pellegrini M, Mao M, Johnson JM: Pathway and gene-set activation measurement from mRNA expression data: the tissue distribution of human pathways. Genome Biol 2006, 7(10):R93. [http://www.ncbi.nlm.nih.gov/pubmed/ 17044931]. [PMID: 17044931]

66. Parts L, Stegle O, Winn J, Durbin R: Joint genetic analysis of gene expression data with inferred cellular phenotypes. PLoS Genet 2011, 7:e1001276. [http://dx.doi.org/10.1371/journal.pgen.1001276]

67. Schadt EE, Lamb J, Yang X, Zhu J, Edwards S, Guhathakurta D, Sieberts SK, Monks S, Reitman M, Zhang C, Lum PY, Leonardson A, Thieringer R, Metzger JM, Yang L, Castle J, Zhu H, Kash SF, Drake TA, Sachs A, Lusis AJ: An integrative genomics approach to infer causal associations between gene expression and disease. Nature Genet 2005, 37:710-717.

doi:10.1186/1471-2105-14-7

Cite this article as: Hänzelmann et al:: GSVA: gene set variation analysis for microarray and RNA-Seq data. BMC Bioinformatics 2013 14:7.

\section{Submit your next manuscript to BioMed Central} and take full advantage of:

- Convenient online submission

- Thorough peer review

- No space constraints or color figure charges

- Immediate publication on acceptance

- Inclusion in PubMed, CAS, Scopus and Google Scholar

- Research which is freely available for redistribution

Submit your manuscript at www.biomedcentral.com/submit
() Biomed Central 